\title{
Hyperbolic Fibre Spaces and Mordell's Conjecture over Function Fields
}

\author{
Dedicated to Professor S. Nakano on his 60 th birthday
}

By

Junjiro NOGUCH"*

\section{Introduction}

Lang [16] discussed the higher dimensional analogue of Mordell's conjecture for curves of genus $\geqq 2$ in terms of hyperbolic manifolds due to $\mathbb{K}$ obayashi [10] and posed a relative formulation of the problem for algebraic families of hyperbolic varieties:

Problem. If there are an infinite number of cross sections, then the family contains split subfamilies, and almost all cross sections ( $i . e_{.}$, all but a finite number of cross sections) are due to constant ones.

Moreover, in the split case, he conjectured the following:

Læm ${ }^{9}$ 's comjecture. Let $\mathbb{M}$ be a projective hyperbolic variety and $\mathbb{N}$ any algebraic variety. Then there are only a finite number of surjective rational mappings of $\mathbb{N}$ onto $M$.

The main purpose of this paper is to give some affirmative answer to the above Problem. We also make clear how Lang's conjecture relates to the above Problem (see (3.1) and the Main Theorem (3.2), (iii) in Section 3). Several finiteness theorems motivated by $\mathbb{L}$ ang's conjecture were obtained by $[15,23,8,28]$. In Section 4 of this

* Department of Mathematics, Tokyo Institute of Technology, Ookayama, Meguroku, Tokyo 152, Japan 
paper we shall show Lang's conjecture for compact hyperbolic Kähler manifolds $M$ with the first Chern class $c_{1}(M) \leqq 0$ and for compact complex spaces $N$, while we do not require $M$ and $N$ to be algebraic (Theorem (4.1)).

Let $(\bar{X}, \bar{\pi} ., \bar{R})$ be a compact normal fibre space over a compact complex space $\bar{R}^{1)}$; i. e., $\bar{X}$ is a compact normal complex space and $\bar{\pi}: \bar{X} \rightarrow \bar{R}$ is a surjective holomorphic mapping of which general fibres $\bar{X}_{t}=\bar{\pi}^{-1}(t)$ with $t \in \bar{R}$ are irreducible. Let $R$ be a Zariski open subset of $\bar{R}, X=\bar{\pi}^{-1}(R)$ and $\pi=\bar{\pi} \mid X$ the restriction of $\bar{\pi}$ over $X$. We say that $(X, \pi, R)$ is a hyperbolic fibre space if $X_{t}=$ $\pi^{-1}(t)$ are hyperbolic for all $t \in R$; that is, the Kobayashi hyperbolic pseudodistances $d_{x_{t}}$ are true distances (cf. $[10,11]$ ). We denote by $\bar{R}_{\text {reg }}$ the set of regular

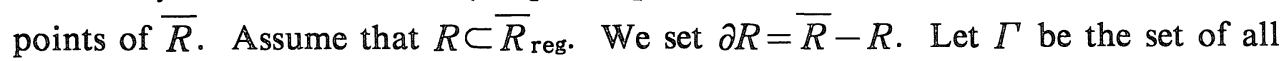
meromorphic cross sections of the fibre space $(X, \pi, R)$ and set $\Gamma(t)=\{s(t) ; s \in$ $\Gamma\}$ for $t \in R$. The main result of this paper is the following:

Main Theorem (3.2). Assume that $(X, \pi, R)$ is a hyperbolic fibre space and that

$$
(X, \pi, R) \text { is hyperbolically imbedded in }(\bar{X}, \bar{\pi}, \bar{R}) \text { along } \partial R
$$

(see Definition (1.3) in Section 1). If there is a point $t_{0} \in R$ such that $\Gamma\left(t_{0}\right)$ is Zariski dense in $X_{t_{0}}$, then there are a compact complex space $\bar{R}^{\prime}$ and a surjective holomorphic mapping $\bar{\lambda}: \bar{R}^{\prime} \rightarrow \bar{R}$ satisfying the following properties:

(i) $\lambda: R^{\prime} \rightarrow R$ is a finitely sheeted unramified covering, where $R^{\prime}=\bar{\lambda}^{-1}(R)$ and $\lambda=\bar{\lambda} \mid R^{\prime}$.

(ii) There is a bimeromorphic mapping $\bar{\Phi}: \bar{R}^{\prime} \times_{R} \bar{X} \rightarrow \bar{R}^{\prime} \times X_{t_{0}}$ such that the restriction $\Phi=\bar{\Phi} \mid R^{\prime} \times{ }_{R} X$ is a holomorphic isomorphism from $\left(R^{\prime} \times{ }_{R} X, \pi^{\prime}, R^{\prime}\right)$ to $\left(R^{\prime} \times X_{t_{0}}, p, R^{\prime}\right)$ as fibre spaces, where $\pi^{\prime}: R^{\prime} \times{ }_{R} X \rightarrow R^{\prime}$ and $p: R^{\prime} \times X_{t_{0}} \rightarrow R^{\prime}$ denote the natural projections.

(iii) Moreover, assume that $X_{t_{0}}$ satisfies property (3.1) (see Section 3) or Aut $\left(X_{t_{0}}\right)=$ $\{1\}$, where $\operatorname{Aut}\left(X_{t_{0}}\right)$ denotes the holomorphic automorphism group of $X_{t_{0}}$. Then we have $\bar{R}^{\prime}=\bar{R}$ and $R^{\prime}=R$.

This Main Theorem (3.2) implies an affirmative answer for the above Problem (see Corollary (3.6) in Section 3). Let $(\bar{X}, \bar{\pi}, \bar{R})$ be a projective algebraic fibre

1) Complex spaces are assumed to be reduced and irreducible, unless otherwise mentioned. 
space defined over an algebraically closed field $k$ of characteristic 0 ; i.e., $\bar{X} \subset \bar{R} \times$ $\mathbb{P}_{k}^{N}$ with the $N$-dimensional projective space $\mathbb{P}_{k}^{N}$ over $k$ and $\bar{\pi}$ is the projection of $\bar{X}$ onto $\bar{R}$. Let $K$ be the function field of $\bar{R}$ and $\mathscr{Q}$ the generic fibre of $(\bar{X}, \bar{\pi}, \bar{R})$, which is a projective variety defined over $K$. Then, assuming the conditions of the Main Theorem (3.2) for $(X, \pi, R)$ and $(\bar{X}, \bar{\pi}, \bar{R})$, we have the following:

If the set $\mathscr{L}(K)$ of $K$-rational points of $\mathscr{X}$ is dense, then there is a finite extension $K^{\prime}$ of $K$ such that $\mathscr{Z} \times_{K} K^{\prime}$ is isomorphic over $K^{\prime}$ to a variety $\mathscr{Z}_{0}$ defined over the constant field $k$.

This is an analogue of Mordell's conjecture for curves over function fields proved by Manin [18] and Grauert [4], who did not assume condition (3.3). Mordell's conjecture for curves over function fields is easily reduced to the case of $\operatorname{dim} R=1$ (see, e. g., $[18,27]$ ). In the case where $\operatorname{dim} X_{t}=\operatorname{dim} R=1$, property (3.1) is nothing but de Franchis' theorem and moreover we see that condition (3.3) is essentially, automatically satisfied (see Theorem (5.2) in Section 5). Therefore, in this case, (iii) of the Main Theorem (3.2) holds and we have $K^{\prime}=K$ in the above assertion.

Some results in this direction were obtained by $[24,22]$. We discuss the relationships among theirs and the present one (see Remark to the Main Theorem (3.2) and Remark to Corollary (3.6) in Section 3).

The proof of the Main Theorem (3.2) is mainly based on the fundamental results of [2] on hyperbolic manifolds (or spaces). In the case of $\operatorname{dim} X_{t}=1$, Green [7] suggested the similar observation to ours and we will discuss it in Section 5.

\section{Acknowledgement}

The author had useful discussions on the subject of Section 5 with Professors M. Suzuki and $\mathbb{T}$. Nishino, to whom he expresses his sincere gratitude.

\section{§ 1. Hyperbolically Imbeddedl Fibre Space}

Let $X$ and $R$ be complex spaces and $\pi: X \rightarrow R$ a proper holomorphic mapping such that general fibres $X_{t}=\pi^{-1}(t)$ with $t \in R$ are irreducible. We call the triple ( $X$, $\pi, R)$ a fibre space. If $X_{t}$ are hyperbolic for all $t \in R$, then we call $(X, \pi, R)$ a hyperbolic fibre space. Here we define the hyperbolicity for reducible connected complex spaces exactly in the same way as in $[10,11]$. We denote by $d_{*}$ the 
Kobayashi hyperbolic pseudodistance of a complex space which may be reducible but connected. Set $X \mid \Delta=\pi^{-1}(\Delta)$ for a subset $\Delta \subset \mathbb{R}$.

Lemma (1.1) ([29, Theorem 4]). Let $(X, \pi, R)$ be a hyperbolic fibre space and $\Delta$ an open connected subset of $R$. If $\Delta$ is (complete) hyperbolic, then $X \mid \Delta$ is (complete) hyperbolic.

Let $T(X)$ denote the Zariski tangent space over $X, h$ a hermitian metric on $X$ and set $\|v\|_{h}=(h(v, \bar{v}))^{1 / 2}$ for $v \in T(X)$. We denote by $F_{X}$ the infinitesimal form of the hyperbolic distance $d_{X}$ (see [25]). Royden [25] proved in general that $X$ is hyperbolic if and only if there is a constant $C>0$ for any compact subset $K \subset X$ such that $F_{X}(v) \geqq C\|v\|_{h}$ for all $v \in T(X) \mid K$. Combining this with Lemma (1.1), we have the following.

Lemma (1.2). Let $(X, \pi, R)$ be a hyperbolic fibre space and $t \in R$. Then there are a neighborhood $U$ of $t$ and a constant $C>0$ such that

$$
F_{X \mid U}(v) \geqq C\|v\|_{h}
$$

for all $v \in T(X \mid U)$.

Let $(\bar{X}, \bar{\pi}, \bar{R})$ be a fibre spàce such that $\bar{R} \supset R, \partial R=\bar{R}-\mathbb{R}$ is a proper analytic subset of $\bar{R}, X=\bar{X} \mid R$ and $\pi=\bar{\pi} \mid X$. If $\bar{X}$ is compact, we say that $(\bar{X}, \bar{\pi}$, $\bar{R})$ is a compactification of $(X, \pi, R)$.

Definition $(1.3)$. We say that the fibre space $(X, \pi, R)$ is hyperbolically imbedded in $(\bar{X}, \bar{\pi}, \bar{R})$ along $\partial R$ if for any point $t \in \partial R$, there is a neighborhood $U \subset \bar{R}$ of $t$ satisfying the following condition :

(1.4) For arbitrary distinct points $x_{1}$ and $x_{2}$ of $\bar{X}_{t}$, there are neighborhoods $V_{i} \subset$ $\bar{X} \mid U$ of $x_{i}, i=1,2$ such that $d_{X \mid(U-\partial R)}\left(V_{1} \cap X, V_{2} \cap X\right)>0$,

where we set

$$
d_{X \mid(U-\partial R)}\left(V_{1} \cap X, V_{2} \cap X\right)=\inf \left\{d_{X \mid(U-\partial R)}\left(y_{1}, y_{2}\right) ; y_{i} \in V_{i}, i=1,2\right\}
$$

It is clear that $(X, \pi, R)$ is hyperbolically imbedded in $(\bar{X}, \bar{\pi}, \bar{R})$ along $\partial R$ if and only if 
(1.5) for an arbitrary point $x \in \bar{X}_{t}$ with any $t \in \partial R$, there are neighborhoods $U$ of $t$ and arbitrarily small $V, W$ of $x$ such that $\bar{V} \subset W$ and $d_{X \mid(U-\partial R)}$ $(V \cap X,(X \mid(U-\partial R))-W)>0$.

We give a similar criterion of the hyperbolic imbeddedness to $[9$, Theorem 1$]$.

Lemma (1. $(\overline{0})$. Let $(X, \pi, R)$ and $(\bar{X}, \bar{\pi}, \bar{R})$ be as above. Then $(X, \pi, R)$ is hyperbolically imbedded in $(\bar{X}, \bar{\pi}, \bar{R})$ along $\partial R$ if and only if for any point $t \in$ $\partial R$ there are a neighborhood $U$ of $t$ in $\bar{R}$ and a constant $C>0$ such that

$$
F_{X \mid(U-\partial R)}(v) \geqq C\|v\|_{h}
$$

for all $v \in T(X \mid(U-\partial R))$.

Proof. The inequality (1.7) obviously implies that $(X, \pi, R)$ is hyperbolically imbedded in $(\bar{X}, \bar{\pi}, \bar{R})$. We show the converse. Assume that it is not true. Then we can take a decreasing sequence $\left\{U_{\nu}\right\}_{\nu=1}^{\infty}$ of neighborhoods of $t$ in $\bar{R}$ and increasing sequence $\left\{r_{\nu}\right\}_{\nu=1}^{\infty}$ of positive real numbers such that $U_{\nu+1}$ are relatively compact in $U_{\nu}, \cap_{\nu=1}^{\infty} U_{\nu}=\{t\}, \lim _{\nu \rightarrow \infty} r_{\nu}=+\infty$ and there are holomorphic mappings $f_{\nu}: D\left(r_{\nu}\right) \rightarrow X \mid\left(U_{\nu}-\partial R\right)$ with $\left\|f_{\nu}^{\prime}(0)\right\|_{h}=1$, where we set $f_{\nu}^{\prime}(z)=f_{\nu *}\left((d / d z)_{z}\right)$ for $z \in D\left(r_{\nu}\right)$. Then by $\left[2\right.$, Lemma 2.1] we have holomorphic mappings $g_{\nu}: D\left(r_{\nu}\right)$ $\rightarrow X \mid\left(U_{\nu}-\partial R\right)$ such that $\left\|g_{\nu}{ }^{\prime}(0)\right\|_{h}=1$ and $\left\|g^{\prime}(z)\right\|_{h} \leqq a_{\nu}(z)$ on $D\left(r_{\nu}\right)$, where

$$
a_{\nu}(z)=\frac{r_{\nu}^{2}}{r_{\nu}^{2}-|z|^{2}}
$$

Since $\left\{a_{\nu}\right\}$ converges to 1 uniformly on compact subsets, $\left\{g_{\nu}\right\}$ is equicontinuous. By Ascoli-Arzela's theorem, we have a subsequence of $\left\{g_{\nu}\right\}$ which converges uniformly on compact subsets to a holomorphic mapping $g: \mathbb{C} \rightarrow X_{t}$. For the simplicity, we denote the subsequence by the same $\left\{g_{\nu}\right\}$. Since $\left\|g_{\nu}{ }^{\prime}(0)\right\|_{h}=1,\left\|g^{\prime}(0)\right\|_{h}=1$, so that $g$ is not a constant mapping. Take $z^{\prime} \in \mathbb{C}$ so that $g\left(z^{\prime}\right) \neq g(0)$, and put

$$
x_{1}=g(0), x_{2}=g\left(z^{\prime}\right) .
$$

We may assume that $r_{\nu}>\left|z^{\prime}\right|$ for $\nu=1,2, \cdots$. Since $\pi \circ g_{\nu}\left(D\left(r_{\nu}\right)\right) \subset U_{\nu}$ and $\lim _{\nu \rightarrow \infty} r_{\nu}$ $=+\infty$, we have for any neighborhood $U$ of $t$ and for any neighborhoods $V_{i}$ of $x_{i}$ in $\bar{X} \mid U, i=1,2$, 


$$
\begin{aligned}
d_{X \mid(U-\partial R)}\left(X \cap V_{1}, X \cap V_{2}\right) & \leqq \lim _{\nu \rightarrow \infty} d_{X \mid(U-\partial R)}\left(g_{\nu}(0), g_{\nu}\left(z^{\prime}\right)\right) \\
& \leqq \lim _{\nu \rightarrow \infty} d_{D\left(r_{\nu}\right)}\left(0, z^{\prime}\right)=0
\end{aligned}
$$

Therefore $(X, \pi, R)$ is not hyperbolically imbedded in $(\bar{X}, \bar{\pi}, \bar{R})$ along $\partial R$.

Q. E. D.

The following is a direct consequence of Lemma (1.6).

Corollary (1.8). The fibre space $(X, \pi, R)$ is hyperbolically imbedded in $(\bar{X}$, $\bar{\pi}, \bar{R})$ if and only if for every point $t \in \partial R$ there are neighborhoods $V$ and $W$ of $t$ such that $V \subset W, V$ is relatively compact in $W, X \mid(W-\partial R)$ is hyperbolic and $X \mid(V-\partial R)$ is hyperbolically imbedded in $\bar{X} \mid W$ (in the sense of $[11, \mathrm{p} .366]$ ).

\section{$\S$ 2. Convergence of Cross Sections}

Let $(X, \pi, R)$ and $(\bar{X}, \bar{\pi}, \bar{R})$ be as in Section 1. In general, a mapping $s$ : $R \rightarrow X$ is called a cross section of the fibre space $(X, \pi, R)$ if $\pi \circ s=\mathrm{id}$. In this section we assume that $(X, \pi, R)$ is a hyperbolic fibre space and hyperbolically imbedded in $(\bar{X}, \bar{\pi}, \bar{R})$ along $\partial R$. We have the following by Corollary (1.8) and [11, Section 3]:

Lemma (2.1). Let $s$ be a meromorphic cross section of $(X, \pi, R)$. Then $s$ extends to a unique meromorphic cross section $\bar{s}$ of $(\bar{X}, \bar{\pi}, \bar{R})$; moreover, if $\bar{R}$ is smooth and if $\partial R$ is of pure codimension 1 and has only normal crossings, then $\bar{s}: \bar{R} \rightarrow \bar{X}$ is a holomorphic cross section of $(\bar{X}, \bar{\pi}, \bar{R})$.

Let $\Gamma$ denote the set of all meromorphic cross sections of $(X, \pi, R)$. In the rest of this section, we assume that $\bar{R}$ is smooth, and that $\partial R$ is of pure codimension 1 and has only normal crossings. By Lemma (2.1) $\Gamma$ coincides with the set of all holomorphic cross sections of $(X, \pi, R)$ and all $s \in \Gamma$ have holomorphic extensions $\bar{s}$ on $\bar{R}$. By Lemma (1.1) and the general theory of hyperbolic spaces (cf. [10]) we see that $\Gamma$ forms a normal family.

Theorem (2.2). The family $\{s ; s \in \Gamma\}$ forms a normal family.

For the proof it suffices to show the following lemma: 
Lemma (2.3). Let the notation be as above. Let $\left\{s_{\nu}\right\}_{\nu=1}^{\infty}$ be a sequence of $s_{\nu}$ $\in \Gamma$ which converges uniformly on compact subsets of $R$ to $s \in \Gamma$. Then the sequence $\left\{s_{\nu}\right\}_{\nu=1}^{\infty}$ converges uniformly on compact subsets of $\bar{R}$ to $\bar{s}$.

Proof. Let $t \in \partial R$ be an arbitrary point. Then there is a relatively compact neighborhood $U$ of $t$ in $\bar{R}$ with holomorphic local coordinates system $\left(z^{1}, \ldots, z^{n}\right)$ $\left(n=\operatorname{dim} R\right.$ ) such that $U$ is biholomorphic to the $n$-dimensional unit polydisc $D^{n}$ $=\left\{\left(z^{i}\right) ;\left|z^{i}\right|<1\right\}, t=(0, \ldots, 0)$ and

$$
\partial R \cap D^{n}=\left\{z^{1 \cdots} z^{k}=0\right\}
$$

for some $1 \leqq k \leqq n$, where we identify $U$ with $D^{n}$. We first consider the case of $k$ $=1$. Therefore $\partial R \cap D^{n}=\left\{z^{1}=0\right\}$ and $R \cap D^{n}=D^{*} \times D^{n-1}$, where $D^{*}=D-\{0\}$. Let $z^{\prime}=\left(z^{2}, \ldots, z^{n}\right) \in D^{n-1}$ and let $O^{\prime}$ be the origin of $D^{n-1}$. We show that $\left\{s_{\nu}\left(z^{1}\right.\right.$, $\left.\left.z^{\prime}\right)\right\}_{\nu=1}^{\infty}$ converges uniformly on compact subsets of $D \times D^{n-1}$ to $\bar{s}\left(z^{1}, z^{\prime}\right)$. Suppose it does not hold. Put $\bar{s}\left(0, O^{\prime}\right)=x$. Then there is a neighborhood $W$ of $x$ such that $W$ is biholomorphic to an analytic subset of the unit $N$-dimensional open ball $B^{N}$ with center at the origin in $\mathbb{C}^{N}$ and $x$ corresponds to the origin. We identify $W$ with the analytic subset of $B^{N}$. By the assumption, for any positive integer $\mu \in \mathbb{N}$ and any $\delta>0$, there are an infinite number of $s_{\nu}$ such that

$$
\bar{s}_{\nu}\left(D\left(\frac{1}{\mu}\right) \times D(\delta)^{n-1}\right) \not \subset W \subset B^{N} .
$$

We denote by $B^{N}(w ; r)$ the open ball of radius $r>0$ with center at $w$ in $\mathbb{C}^{N}$ and put $B^{N}(r)=B^{N}(O ; r)$. There are $\mu_{0} \in \mathbb{N}$ and $0<\delta_{0}<1$ such that $\bar{s}\left(\overline{D\left(1 / \mu_{0}\right.}\right)$ $\left.\times D\left(\delta_{0}\right)^{n-1}\right) \subset B^{N}(1 / 8)$, where $\overline{D\left(1 / \mu_{0}\right)}$ is the closure of $D\left(1 / \mu_{0}\right)$. Let $\partial D(1 / \mu)=$ $\overline{D(1 / \mu)}-D(1 / \mu)$ be the boundary circle of $D(1 / \mu)$. Since $\left\{s_{\nu}\right\}_{\nu}$ converges uniformly on $\partial D(1 / \mu) \times D\left(\delta_{0}\right)^{n-1}$ to $\bar{s}$, we can choose a subsequence $\left\{s_{\nu_{\mu}}\right\}_{\mu=1}^{\infty}$ of $\left\{s_{\nu}\right\}_{\nu}$ and a sequence $\left\{z_{\mu}^{\prime}\right\}_{\mu=1}^{\infty}$ of $z_{\mu}^{\prime} \in D\left(\delta_{0}\right)^{n-1}$ such that $\lim _{\mu \rightarrow \infty} z_{\mu}^{\prime}=O^{\prime}$ and

$$
\left\{\begin{array}{l}
\bar{s}_{\nu_{\mu}}\left(\partial D(1 / \mu), z_{\mu}^{\prime}\right) \subset B^{N}(1 / 4), \\
\bar{s}_{\nu_{\mu}}\left(D(1 / \mu), z_{\mu}^{\prime}\right) \not \subset B^{N} .
\end{array}\right.
$$

Hence there are points $z_{\mu}^{1} \in D(1 / \mu), \mu=1,2, \ldots$ such that

$$
x_{\mu}=\bar{s}_{\nu_{\mu}}\left(z_{\mu}^{1}, z_{\mu}^{\prime}\right) \in \partial B^{N}\left(\frac{1}{2}\right) .
$$


Put $\Sigma_{\mu}=\bar{s}_{\nu_{\mu}}\left(D(1 / \mu), z_{\mu}^{\prime}\right) \cap B^{N}\left(x_{\mu} ; 1 / 8\right)$. Then by $(2.4), \Sigma_{\mu}$ are 1-dimensional analytic subvarieties of $B^{N}\left(x_{\mu} ; 1 / 8\right)$. Thus the euclidean volumes $\operatorname{Vol}\left(\Sigma_{\mu}\right)$ of $\Sigma_{\mu}$ in $B^{N}\left(x_{\mu} ; 1 / 8\right)$ satisfy

$$
\operatorname{Vol}\left(\Sigma_{\mu}\right) \geqq \frac{\pi}{64}, \quad \mu=1,2, \cdots
$$

Let $h$ be a hermitian metric on $\bar{X}$ and $\operatorname{Vol}_{h}\left(\Sigma_{\mu}\right)$ denote the volumes of $\Sigma_{\mu}$ with respect to $h$. Then there is a positive constant $C_{1}$ such that

$$
\operatorname{Vol}_{h}\left(\Sigma_{\mu}\right) \geqq C_{1}>0, \quad \mu=1,2, \cdots
$$

Let $H_{h}^{2}$ (resp. $H_{F_{X \mid D^{*} \times D^{n-1}}}^{2}$ ) denote the 2-dimensional Hausdorff measure defined by $h$ (resp. $F_{X \mid D^{*} \times D^{n-1}}$ ). Then there is a positive constant $C_{2}$ such that

$$
\operatorname{Vol}_{h}\left(\Sigma_{\mu}\right) \leqq C_{2} H_{h}^{2}\left(\Sigma_{\mu}\right), \mu=1,2, \cdots
$$

Put $y_{\mu}=\bar{s}_{\nu \mu}\left(0, z_{\mu}^{\prime}\right)$ and $\Sigma_{\mu}^{*}=\Sigma_{\mu}-\left\{y_{\mu}\right\}$. Then we have that $H_{h}^{2}\left(\Sigma_{\mu}\right)=H_{h}^{2}\left(\Sigma_{\mu}^{*}\right)$. We may assume that $U$ is chosen so that Lemma (1.6) holds. Hence there is a positive constant $C_{3}$ such that

$$
H_{h}^{2}\left(\Sigma_{\mu}\right)=H_{h}^{2}\left(\Sigma_{\mu}^{*}\right) \leqq C_{3} H_{F_{X \mid D^{*} \times D^{n-1}}^{2}}\left(\sum_{\mu}^{*}\right), \mu=1,2, \cdots
$$

Let $\alpha$ denote the Poincare metric on $D^{*}$ and $H_{\alpha}^{2}$ the 2-dimensional Hausdorff measure defined by $\alpha$. Then we have by the decreasing property of hyperbolic distances for holomorphic mappings that

$$
H_{F_{X \mid D^{*} \times D^{n-1}}^{2}}\left(\sum_{\mu}^{*}\right) \leqq H_{\alpha}^{2}\left(D^{*}\left(\frac{1}{\mu}\right)\right) \leqq \frac{C_{4}}{\log \mu}, \quad \mu=1,2, \cdots
$$

where $C_{4}$ is a positive constant. It follows from (2.6)-(2.9) that

$$
0<C_{1} \leqq \frac{C_{2} C_{3} C_{4}}{\log \mu} \rightarrow 0 \text { as } \mu \rightarrow \infty
$$

This is a contradiction.

Now we consider the general case of $k \geqq 1$. Then we have that $D^{n}-\partial R=\left(D^{*}\right)^{k}$ $\times D^{n-k}$. Applying the above result, we infer that $\left\{s_{\nu}\right\}_{\nu}$ converges uniformly on compact subsets of $\left(D^{*}\right)^{k-1} \times D^{n-k+1}$ to $\bar{s}$. Thus the induction on $k$ completes the proof.

Q.E.D. 


\section{§3。 Maim Theorem}

Let $(\bar{X}, \bar{\pi}, \bar{R})$ be a compact fibre space and a compactification of a fibre space $(X, \pi, R)$. Assume that $X$ is normal and $R \subset \bar{R}_{\text {reg. }}$ Let $\Gamma$ denote the set of meromorphic cross sections of $(X, \pi, R)$ and put

$$
\Gamma(t)=\{s(t) ; s \in \Gamma\}
$$

for $t \in \mathbb{R}$.

Relating to Lang's conjecture in Introduction, we consider, in general, the following property for a compact complex space $Y$ :

(3.1) For any compact complex space $N$, there are only a finite number of surjective meromorphic mappings from $N$ onto $Y$.

See the next Section 4 for several sufficient conditions for $Y$ which imply property (3.1).

$\mathbb{M}$ IIm Theorem (3.2D。. Assume that $(X, \pi, \mathbb{R})$ is a hyperbolic fibre space and that

$$
(X, \pi, R) \text { is hyperbolically imbedded in }(\bar{X}, \bar{\pi}, \bar{R}) \text { along } \partial R \text {. }
$$

If there is a point $t_{0} \in R$ such that $\Gamma\left(t_{0}\right)$ is Zariski dense in $X_{t_{0}}$, then there are a compaci complex space $\bar{R}^{\prime}$ and a holomorphic mapping $\bar{\lambda}: \bar{R}^{\prime} \rightarrow \bar{R}$ satisfying the followings:

(i) $\lambda: R^{\prime} \rightarrow R$ is a finitely sheeted unramified covering, where $\mathbb{R}^{\prime}=\bar{\lambda}^{-1}(R)$ and $\lambda=\bar{\lambda} \mid \mathbb{R}^{\prime}$ 。

(ii) There is a Bimeromorphic mapping $\bar{\Phi}: \bar{R}^{\prime} \times{ }_{R} \bar{X} \rightarrow \bar{R} \times X_{t_{0}}$ such that the restriction $\bar{\Phi} \mid \mathbb{R}^{\prime} \times_{R} X$ is a holomorphic isomorphism from $\left(\mathbb{R}^{\prime} \times_{R} X, \pi^{\prime}, \mathbb{R}^{\prime}\right)$ to $\left(R^{\prime} \times\right.$ $\left.X_{t_{0}}, p, R^{\prime}\right)$ as fibre spaces, where $\pi^{\prime}: \mathbb{R}^{\prime} \times_{R} X \rightarrow R^{\prime}$ and $p: R^{\prime} \times X_{t_{0}} \rightarrow R^{\prime}$ denote the first projections.

(iii) Moreover, assume that $X_{t_{0}}$ satisfies (3.1) or $\operatorname{Aut}\left(X_{t_{0}}\right)=\{1\}$, where $\operatorname{Aut}\left(X_{t_{0}}\right)$ denotes the holomorphic automorphism group of $X_{i_{0}}$. Then we have $\bar{R}^{\prime}=\bar{R}$ and $\mathbb{R}^{\prime}=\mathbb{R}$.

Remark. In the previous paper [22] we proved a similar result to the Main Theorem (3.2) in the case where $(X, \pi, R)$ is an algebraic smooth fibre space and 
the tangent bundle $T\left(X_{t}\right)$ is negative (see, e.g., [22]) for generic $t \in R$, while we did not assume any condition such as (3.3). If $T\left(X_{t}\right)$ is negative, then $X_{t}$ is hyperbolic and satisfies property $(3.1)$ (see $[12,15,23,28,8]$ ).

We first reduce the Main Theorem (3.2) to the case where $\bar{R}$ is smooth, $\partial R$ is of pure codimension 1 and has only normal crossings, and $\bar{X}$ is normal. Assume the assumptions in the Main Theorem (3.2). By Hironaka's theorem, there is a desingularization $\alpha: \bar{R}_{0} \rightarrow \bar{R}$ such that $\bar{R}_{0}$ is smooth and $\bar{R}_{0}-\alpha^{-1}(R)$ has pure codimension 1 and only normal crossings. Put $R_{0}=\alpha^{-1}(R)$. Then $\alpha \mid R_{0}: R_{0} \rightarrow R$ is biholomorphic. Put

$$
\bar{X}_{0}^{\prime}=\bar{R}_{0} \times_{\bar{R}} \bar{X}, X_{0}^{\prime}=R_{0} \times_{R} X .
$$

Let $\bar{\pi}_{0}^{\prime}: \bar{X}_{0} \rightarrow \bar{R}_{0}$ and $\pi_{0}: X_{0}^{\prime} \rightarrow R^{\prime}$ be the natural projections. Let $\beta: \bar{X}_{0} \rightarrow \bar{X}_{0}^{\prime}$ be the normalization of $\bar{X}_{0}^{\prime}$. Since $X_{0}^{\prime}$ is biholomorphic to $X$ and $X$ is normal, $X$ is biholomorphic to $\beta^{-1}\left(X_{0}^{\prime}\right)$, which is denoted by $X_{0}$. Put $\bar{\pi}_{0}=\bar{\pi}_{0}{ }^{\prime} \circ \beta$ and $\pi_{0}=$ $\bar{\pi}_{0} \mid X_{0}$. We easily obtain the following:

Lemma (3.4) Let the notation be as above. Then the fibre space $\left(X_{0}, \pi_{0}, R_{0}\right)$ is hyperbolic and hyperbolically imbedded in $\left(\bar{X}_{0}, \bar{\pi}_{0}, \bar{R}_{0}\right)$ along $\partial R_{0}$.

Proof of Main Theorem (3.2). By Lemma (3.4) we may assume that $\bar{R}$ is smooth and that $\partial R$ has pure codimension 1 and only normal crossings. Then, by Lemma (2.1) all $s \in \Gamma$ are holomorphic and have holomorphic extensions on $\bar{R}$. Therefore $\Gamma$ is identified with the set of holomorphic cross sections of $(\bar{X}, \bar{\pi}, \bar{R})$. We endow $\Gamma$ with the compact-open topology. It follows from Theorem (2.2) that $\Gamma$ is compact. By [3] $\Gamma$ carries a structure of complex space such that the mapping

$$
\Psi: \bar{R} \times \Gamma \ni(t, s) \rightarrow s(t) \in \bar{X}
$$

is holomorphic. We show that there is an irreducible component $\Gamma_{0}$ of $\Gamma$ such that $\Psi\left(\bar{R} \times \Gamma_{0}\right)=\bar{X}$. Assume that there is no such $\Gamma_{0}$. Take any irreducible component $\Gamma_{1}$ of $\Gamma$ and set $\Gamma_{1}(t)=\left\{s(t) ; s \in \Gamma_{1}\right\}$ for $t \in \bar{R}$. Then we have

$$
\operatorname{dim} \Gamma_{1}\left(t_{0}\right)=\operatorname{dim} \Psi\left(\bar{R} \times \Gamma_{1}\right)-\operatorname{dim} \bar{R}<\operatorname{dim} X-\operatorname{dim} R \leqq \operatorname{dim} X_{t_{0}}
$$

Hence $\Gamma_{1}\left(t_{0}\right)$ is a proper analytic subset of $X_{t_{0}}$, so that $\Gamma\left(t_{0}\right)$ is a proper analytic subset of $X_{t_{0}}$. This is absurd. We denote $\Psi \mid \bar{R} \times \Gamma_{0}$ by the same letter $\Psi$. Thus we get a surjective holomorphic mapping 


$$
\Psi: \bar{R} \times \Gamma_{0} \ni(t, s) \mapsto s(t) \in \bar{X} .
$$

Let $T(\bar{X})$ (resp. $T(\bar{R})$ ) denote the Zariski tangent space over $\bar{X}$ (resp. $\bar{R}$ ). Set $\widetilde{T}(\bar{R})=\bar{\pi}^{-1} T(\bar{R})$ and let $\tilde{\pi}_{*}: T(\bar{X}) \rightarrow \widetilde{T}(\bar{R})$ be the morphism induced by $\bar{\pi}_{*}: T(\bar{X}) \rightarrow T(\bar{R})$. Set

$$
F=\bigcup_{x \in \bar{X}}\left\{\sigma_{x} \in \mathbb{H o m}\left(\widetilde{T}(\bar{R})_{x}, T(\bar{X})_{x}\right) ; \tilde{\pi}_{*}^{\circ} \sigma_{x}=\mathrm{id}\right\} .
$$

Then $F$ naturally carries a structure of a complex space and the natural projection $q: F \rightarrow \bar{X}$ is holomorphic. Put $F_{x}=q^{-1}(x)$ for $x \in \bar{X}$. The differential $s_{*}(t)$ : $T(\bar{R})_{t} \rightarrow T(\bar{X})_{s(t)}$ of $s \dot{\epsilon} \Gamma_{0}$ naturally defines an element $\widetilde{s}_{*}(t) \in F_{s(t)}$. We set

$$
\Psi^{\prime}: \bar{R} \times \Gamma_{0} \ni(t, s) \mapsto \widetilde{S}_{*}(t) \in F,
$$

which is a holomorphic mapping. Since $\Gamma_{0}$ is compact and $F_{x}$ is an analytic subset of some $\mathbb{C}^{N}, \Psi^{\prime}\left(\bar{\pi}(x), \Gamma_{0}\right) \cap F_{x}$ is a finite set. Putting $E=\Psi^{\prime}\left(\bar{R} \times \Gamma_{0}\right)$, we have a finite cover over $\bar{X}$

$$
q \mid E: E \rightarrow \bar{X}
$$

which may be ramified. Let $k$ be the number of sheets of the cover $q \mid E: E \rightarrow \bar{X}$ and set

$$
\eta_{x}=\frac{1}{k} \sum_{\sigma \in(q \mid E)^{-1}(x)} \sigma
$$

for $x \in \bar{X}$, where the sum is taken with counting multiplicities. Let $v$ be a holomorphic vector field on an open subset $U \subset \bar{R}$. Then $v$ naturally defines a holomorphic cross section $\widetilde{v} \in H^{0}(\bar{X} \mid U, \widetilde{T}(\bar{R}))$. Here one notes that $\widetilde{T}(\bar{R})$ is a holomorphic vector bundle. Therefore $\eta_{x}(\widetilde{v}) \in T(\bar{X})_{x}$ for $x \in \bar{X} \mid U$ and

$$
\eta(\widetilde{v}): \bar{X}_{\mathrm{reg}} \cap(\bar{X} \mid U) \ni x \mapsto \eta_{x}(\widetilde{v}) \in T(\bar{X})
$$

is a holomorphic vector field. Since $\bar{X}$ is normal, $\eta(\widetilde{v})$ is extended as a holomorphic vector field on $\bar{X} \mid U$. By definition we have

$$
\bar{\pi}_{* \circ} \eta(\widetilde{v})=v .
$$

Therefore $\eta$ defines the holomorphic horizontal direction field in the fibre space $(\bar{X}$, $\bar{\pi}, \bar{R})$. We see by the general theory of fibre spaces that $(\bar{X}, \bar{\pi}, \bar{R})$ is a fibre bundle with typical fibre $X_{t_{0}}$ and with structure group $\operatorname{Aut}\left(X_{t_{0}}\right)$ such that for a sufficiently 
small neighborhood $U$ of a point $t \in \bar{R}$, there is a trivialization $\bar{X} \mid U \cong U \times X_{t_{0}}$ and $\eta(\widetilde{v})=v \oplus O \in T(U) \oplus T\left(X_{t_{0}}\right) \cong T(\bar{X} \mid U)$ for $v \in T(U)$. It follows from [26] that the bundle $(\bar{X}, \bar{\pi}, \bar{R})$ comes from a monodromy $\rho \in \mathbb{H}$ om $\left(\pi_{1}(\bar{R})\right.$, Aut $\left.\left(X_{t_{0}}\right)\right)$, where $\pi_{1}(\bar{R})$ denotes the fundamental group of $\bar{R}$. Since $\operatorname{Aut}\left(X_{t_{0}}\right)$ is finite ([11, Theorem 9.2]), we have a finite unramified covering $\bar{\lambda}: \bar{R}^{\prime} \rightarrow \bar{R}$ such that there is a holomorphic isomorphism $\bar{\Phi}$ from the fibre space $\left(\bar{R}^{\prime} \times \bar{R} \bar{X}, \bar{\pi}^{\prime}, \bar{R}^{\prime}\right)$ onto $\left(\bar{R} \times X_{t_{0}}\right.$, $\left.\bar{p}, \overline{R^{\prime}}\right)$, where $\bar{\pi}^{\prime}: \bar{R}^{\prime} \times \bar{R} \bar{X} \rightarrow \overline{R^{\prime}}$ and $\bar{p}: \bar{R}^{\prime} \times X_{t_{0}} \rightarrow \bar{R}^{\prime}$ are the natural projections. Therefore we have proved (i) and (ii).

If $\operatorname{Aut}\left(X_{t_{0}}\right)=\{1\}$, then $\rho$ is trivial, so that $(\bar{X}, \bar{\pi}, \bar{R})$ is globally trivial. Assume that $X_{t_{0}}$ satisfies (3.1). Let $U$ be an arbitrary simply connected subdomain of $\bar{R}$. Then we have a trivialization, $\bar{X} \mid U \cong U \times X_{t_{0}}$. The holomorphic mapping $\Psi^{\prime} \mid U \times \Gamma_{0}: U \times \Gamma_{0} \rightarrow U \times X_{t_{0}}$ is written in the form

$$
\Psi \mid U \times \Gamma_{0}(t, s)=(t, s(t)) \in U \times X_{t_{0}}
$$

Set $\Psi \mid U \times \Gamma_{0}(t, s)=\left(t, \phi_{t}(s)\right) \in U \times X_{t_{0}}$ for $(t, s) \in U \times \Gamma_{0}$. Then $\phi_{t}: \Gamma_{0} \ni s$ $\mapsto \phi_{t}(s) \in X_{t_{0}}$ are holomorphic surjections for all $t \in U$. It follows from property (3.1) that $\psi_{t}$ is constant in $t$. We denote it by $\psi$. Thus we have

$$
\Psi \mid U \times \Gamma_{0}(t, s)=(t, \psi(s)) \in U \times X_{t_{0}} .
$$

Hence all $s \in \Gamma_{0}$ are locally constant cross sections and $\eta_{s(t)}=\widetilde{s}_{s}(t)$ for $s \in \Gamma_{0}$ and $t$ $\in \bar{R}$. Thus we deduce that $\rho$ is trivial and then $(\bar{X}, \bar{\pi}, \bar{R})$ is globally trivial.

Q.E.D.

By the above proof we have the following corollaries.

Corollary (3.5)。 If $\bar{R}$ is smooth and $\partial R$ has only normal crossings in the Main Theorem (3.2), then $\bar{\lambda}: \bar{R}^{\prime} \rightarrow \bar{R}$ is an unramified Galois covering and $\bar{\Phi}$ is a holomorphic isomorphism between the fibre spaces.

Corollary (3.6). Let the notation be as in the Main Theorem (3.2). Assume that $\bar{X}$ and $\bar{R}$ are algebraic varieties and that $\Gamma$ is infinite. Then there is a fibre subspace $(\bar{Y}, \bar{\pi} \mid \bar{Y}, \bar{R})$ of $(\bar{X}, \bar{\pi}, \bar{R})$ (i. e., $\bar{Y}$ is an irreducible complex subspace of $\bar{X}$ and $(\bar{Y}, \bar{\pi} \mid \bar{Y}, \bar{R})$ is a fibre space) satisfying the following properties:

(i) $\bar{Y}_{t}=\bar{\pi}^{-1}(t)$ are irreducible curves for $t \in \bar{R}$. 
(ii) Let $\alpha: \bar{Y}^{\prime} \rightarrow \bar{Y}$ be the normalization of $\bar{Y}$ and set $\bar{\pi}^{\prime}=\bar{\pi} \circ \alpha, Y^{\prime}=$ $\bar{\pi}^{\prime-1}(R)$ and $\pi^{\prime}=\bar{\pi}^{\prime} \mid Y^{\prime}$. Then there is a bimeromorphic mapping $\bar{\varphi}$ from $\bar{Y}^{\prime}$ onto $\bar{R} \times Y_{t}^{\prime}$ with some $t \in R$, such that $\varphi=\bar{\varphi} \mid Y^{\prime}$ is a holomorphic isomorphism of $\left(Y^{\prime}, \pi^{\prime}, R\right)$ onto $\left(R \times Y_{t}^{\prime}, p_{1}, R\right)$ as fibre spaces, where $p_{1}: R \times Y_{t}^{\prime} \rightarrow R$ is the natural projection.

(iii) $\Gamma$ contains an infinite number of rational cross sections of $(\bar{Y}, \bar{\pi} \mid \bar{Y}, \bar{R})$ and hence of $\left(\bar{Y}^{\prime}, \bar{\pi}^{\prime}, \bar{R}\right)$, all of which, except for a finite number of ones, are due to constant cross sections of $\left(\mathbb{R} \times Y_{t}^{\prime}, p_{1}, R\right)$.

Proof. By the proof of the Main Theorem (3.2), $\Gamma$ is a compact algebraic variety of positive dimension which may be reducible. One can find a compact irreducible curve $C \subset \Gamma$ such that the dimension of the image of the holomorphic mapping

$$
\Psi: \bar{R} \times C \ni(t, s) \mapsto s(t) \in \bar{X}
$$

is $\operatorname{dim} R+1$. Set $\bar{Y}=\Psi(\bar{R} \times C)$. Then $\bar{Y}$ and $\bar{Y}_{t}=\Psi(t, C)$ are irreducible. Put $Y=(\bar{\pi} \mid \bar{Y})^{-1}(R)$. Then $(Y, \pi \mid Y, R)$ is a hyperbolic fibre space and hyperbolically imbedded in $(\bar{Y}, \bar{\pi} \mid \bar{Y}, \bar{R})$ along $\partial R$. Hence our assertions easily follow from the Main Theorem (3.2) and de Franchis' theorem (cf. Section 4, a)). Q.E.D.

Remark. Riebesehl [24, Satz 6.3] proved Corollary (3.6) in the case where $R$ $=\bar{R}, R$ is a smooth curve, $X \subset R \times \mathbb{P}^{N}(\mathbb{C}), \pi$ is the restriction over $X$ of the projection from $R \times \mathbb{P}^{N}(\mathbb{C})$ to $R$ and

every fibre $X_{t}$ with $t \in R$ carries a differential metric with strictly negative curvature.

(See [24, Section 1].) By [12] the condition (3.7) implies that $X_{t}$ is hyperbolic, and property (3.1) holds for such $X_{t}$ (see $[8,28]$ ).

Example. Let $A$ be a simple Abelian variety of dimension $n$ and $C$ an algebraic curve contained in $A$. Let $A \subset \mathbb{P}^{N}(\mathbb{C})$ be an imbedding such that there is no hyperplane containing $A$ and there are hyperplanes $H_{1}, \ldots, H_{n-1}$ such that $H_{1} \cap$ $\cdots \cap H_{n-1} \cap A \supset C$. Let $\mathbb{P}^{N}(\mathbb{C})^{*}$ be the dual projective space of $\mathbb{P}^{N}(\mathbb{C})$ and $\langle H\rangle$ denotes the corresponding point of a hyperplane $H \subset \mathbb{P}^{N}(\mathbb{C})$. Let $R$ be the linear subspace in $\mathbb{P}^{N}(\mathbb{C})^{*}$ generated by $\left\langle H_{1}\right\rangle, \ldots,\left\langle H_{n-1}\right\rangle, X=U_{\langle H\rangle \in R}\{\langle H\rangle\} \times(H \cap A)$ 
$\subset R \times A$ and $\pi: X \rightarrow R$ the natural projection. Since any proper subvariety of $A$ is hyperbolic ([6, Theorem 1$]),(X, \pi, R)$ is a compact hyperbolic fibre space and contains a trivial subfamily $C \times R$. One notes that $X_{t}$ are of general type for $t \in R$ and hence satisfy (3.1) (cf. Section $4, \mathrm{~b})$ ). Let $\Gamma$ be the set of all rational cross sections of $(X, \pi, R)$. We show that $\Gamma(t)$ is not Zariski dense in $X_{t}$ for any $t \in$ $R$. Assume that there is such a point $t_{0} \in R$. Then by the Main Theorem (3.2) we have an isomorphism

$$
\Phi: R \times X_{t_{0}} \rightarrow X \subset R \times A \text {. }
$$

We set $\Phi(t, x)=(t, \varphi(t, x)) \in R \times A$. We have a holomorphic mapping $R \ni$ $t \rightarrow \varphi(t, x) \in A$ for every fixed $x \in X_{t_{0}}$. Since $R=\mathbb{P}^{n-2}(\mathbb{C}), \varphi(t, x)$ is constant in $t$. This is a contradiction.

Here one notes that the metric on a curve in $A$ induced by the flat one does not necessarily have the strictly negative Gaussian curvature. For instance, let $C$ be a hyperelliptic curve of genus 3 of which Jacobian variety $A$ is simple. By comparing the dimensions of moduli spaces of hyperelliptic curves with that of polarized Abelian varieties which are not simple, one easily sees the existence of such $C$. Then by [17], the Gaussian curvature of the metric on $C$ induced by the flat one on $A$ vanishes at the Weierstrass points of $C$. (Unfortunately, the remark of $[16$, p. 780] is false, but Green [6] proved the hyperbolicity of a proper subvariety of a simple complex torus by a different method.)

\section{§ 4. Lang's Conjecture and Property (3.1)}

In the Main Theorem (3.2) we considered property (3.1) which is related to Lang's conjecture in Introduction. It is conjectured that compact hyperbolic spaces will satisfy (3.1). We first recall several known results on sufficient conditions implying (3.1) from the view point of hyperbolic manifolds.

a ) The case of curves. Let $C$ be a compact complex algebraic curve. Then $C$ is hyperbolic if and only if the genus of $C$ is greater than 1 ; in this case, property (3.1) is nothing but de Franchis' theorem.

b ) The case of higher dimension. Let $M$ be a compact complex manifold. Kobayashi and Ochiai [15] proved that if $M$ is of general type (cf. [15]), then there 
are only finitely many surjective meromorphic mappings from any compact complex manifold onto $M$.

c) The case of surfaces. It is recently proved by [19] that a complex projective smooth surface is of general type if and only if it is measure hyperbolic in the sense of $[10$, Chap. IX]. In general, hyperbolic spaces are measurehyperbolic. Combining these with the above $\mathbb{K}$ obayashi-Ochiai's result $b$ ), one sees that complex projective hyperbolic surfaces satisfy (3.1).

See $[8,15,23,28]$ for the other related results.

In this section we show the following partial answer to Lang's conjecture, while manifolds are not assumed to be algebraic but $\mathbb{K}$ ähler.

Theorem (4.11). Let $M$ be a compact hyperbolic $\mathbb{K}$ ähler manifold with $c_{1}(\mathbb{M})$ $\leqq 0$, and $N$ a compact complex space. Then there are only finitely many surjective meromorphic mappings from $N$ onto $M$.

Proof. By Hironaka's theorem on desingularization, we may assume that $N$ is smooth. Then all meromorphic mappings from $N$ into $M$ is holomorphic ([11, Section 3]). Let $\operatorname{Hol}(N, \mathbb{M})$ be the set of all holomorphic mappings from $N$ into $M$ endowed with compact-open topology. By [3] $\operatorname{Hol}(N, M)$ carries a structure of complex space such that the mapping

$$
\operatorname{Hol}(N, M) \times N \ni(f, x) \mapsto f(x) \in M
$$

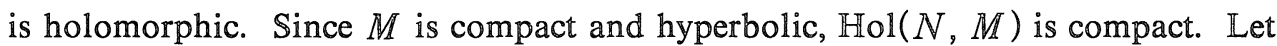
$\Xi$ be the complex subspace of all $f \in \operatorname{Hol}(N, M)$ such that $f(N)=M$. It is noted that $\Xi$ is open and closed in $\operatorname{Hol}(N, M)$. We show that $\operatorname{dim} \Xi=0$. Assume that $\operatorname{dim} \Xi>0$. Then we have a holomorphic mapping $f \in \Xi$ and a nontrivial infinitesimal deformation $\sigma \in H^{0}\left(N, f^{-1} T(M)\right)$ of $f$, so that $p \circ q \circ \sigma=f$, where $q: f^{-1} T(M) \rightarrow T(M)$ is the natural homomorphism induced by $f$, and $p$ : $T(M) \rightarrow M$ is the projection. Set $Z=q \circ \sigma(N)$. Then $Z$ is a compact complex subspace of $T(M)$ different to the zero-section, and $p \mid Z: Z \rightarrow M$ is surjective. Therefore $p \mid Z: Z \rightarrow M$ is a finite ramified covering, of which sheet number is denoted by $l$. Taking into account the elementary symmetric polynomials of degree $l$ in $y_{1}, \ldots, y_{l} \in(p \mid Z)^{-1}(x)$ for general $x \in M$, we find a non-zero holomorphic cross section $\tau \in H^{0}\left(M, S^{l} T(M)\right)$, where $S^{l} T(M)$ denotes the $l$-th symmetric 
tensor power of $T(M)$. Since $M$ is hyperbolic, the universal covering $\widetilde{M}$ of $M$ is hyperbolic, so that there is no euclidean factor in the de $\mathbb{R h a m}$ decomposition of $\widetilde{M}$ (cf. [14, Chap. IX, Section 8]). By [13, Theorem 7] we see that $H^{0}\left(M, S^{l} T(M)\right)=$ $\{O\}$, while $\tau \neq 0$. This is a contradiction.

Q.E.D.

\section{§5. Remarks to the Case of dim $X_{t}=1$}

By making use of the method of [2], Green [5] and A. Howard independently proved the following theorem:

Theorem A. Let $M$ be a compact complex manifold and $D$ a union of irreducible hypersurfaces $D_{1}, \ldots, D_{l}$ which may be singular. Then either

(i) $M-D$ is complete hyperbolic and hyperbolically imbedded in $\mathbb{M}$,

(ii) there is a non-constant holomorphic mapping $f: \mathbb{C} \rightarrow \mathbb{M}-\mathbb{D}$, or

(iii) there is a non-constant holomorphic mapping $f: \mathbb{C} \rightarrow \mathbb{D}_{i_{1}} \cap \cdots \cap \mathbb{D}_{i_{k}}$ $\left(D_{i_{k+1}} \cup \cdots \cup D_{i_{l}}\right)$ for some choice of indices $\left\{i_{1}, \ldots, i_{l}\right\}=\{1, \ldots, l\}$.

Applying this Theorem $\mathbb{A}$ or more precisely the arguments of the proof, Green [7] claims the following:

(5.1) Let $\gamma: W \rightarrow D^{3 g-3}$ be a local universal deformation of stable curves of genus $g \geqq 2$ and $S(W)$ denote the set of singular fibres in $W$. Then $(W-S(W)$, $\left.\gamma \mid(W-S(W)), D^{3 g-3}-\gamma(S(W))\right)$ is hyperbolically imbedded in (W, $\gamma$, $\left.D^{3 g-3}\right)$ along $\gamma(S(W))$.

Then he [7] suggested a proof of Mordell's conjecture for curves over function fields (cf. $[18,4]$ ).

In general, $W$ may have singularities, while the fact that $D_{i}$ are locally principal hypersurfaces was crucial in the proof of Theorem $\mathbb{A}$. It seems to be unknown if the claim (5.1) holds in its form. Here we show it in the case where the base space (the parameter space) is 1-dimensional. Mordell's conjecture for curves over function fields is easily reduced to the case where function fields have transcendental degree 1 (see $[18,27]$ ). Therefore, to get a proof of that conjecture, it is sufficient to consider this case.

Let $\bar{R}$ be a smooth irreducible curve and $R$ a non-empty Zariski open subset of $\bar{R}$. Then $\partial R$ is a finite set of points of $\bar{R}$. Let $(X, \mu, R)$ be a fibre space such 
that $X$ is smooth, $X_{t}$ are smooth irreducible curves of genus $g \geqq 2$ for all $t \in \mathbb{R}$.

Theorem (5.2). Let the notation be as above. Then there is a compactification $(\bar{X}, \bar{\mu}, \bar{R})$ of $(X, \mu, \mathbb{R})$ such that $(X, \mu, R)$ is hyperbolically imbedded in $(\bar{X}, \bar{\mu}, \bar{R})$ along $\partial R$.

Localizing the problem around the points of $\partial R$, we let $\left(Z, \mu, D^{*}\right)$ be a fibre space such that $Z$ is a 2 -dimensional complex manifold, $\mu$ is everywhere of rank 1 and $Z_{t}$ are smooth irreducible curves of genus $g \geqq 2$ for all $t \in D^{*}$. For the proof of Theorem (5.2) it suffices to show the following lemma.

Lemma (5.3)。 Let $\left(Z, \mu, D^{*}\right)$ be as above. Then there is a fibre space $(\bar{Z}$, $\bar{\mu}, D)$ such that $\bar{Z}\left|D^{*}=Z, \bar{\mu}\right| Z=\mu$ and $\left(Z, \mu, D^{*}\right)$ is hyperbolically imbedded in $(\overline{\mathbb{Z}}, \bar{\mu}, \mathbb{D})$ along $\partial D^{*}=\{0\}$.

Proof. We put $\vartheta_{l}: D \ni z \mapsto z^{l} \in \mathbb{D}$ for a positive integer $l \in \mathbb{N}$. By [20, Sections 1 and 3] there are some $l \in \mathbb{N}$ and fibre spaces $(\bar{Z}, \bar{\mu}, D)$ and $\left(\bar{Z}^{\prime}, \overline{\mu^{\prime}}, D\right)$ such that

i) $\mathbb{Z}=\bar{Z} \mid D^{*}$ and $\mu=\bar{\mu} \mid \mathbb{Z}$,

ii ) $\left(\bar{Z}^{\prime}, \bar{\mu}^{\prime}, D\right)$ is the lifting of $(\bar{Z}, \bar{\mu}, D)$ by $\vartheta_{l}$,

iii) $\bar{Z}^{\prime}$ is normal,

iv) the order of any irreducible component of $\bar{Z}_{0}^{\prime}$ is 1 ,

v) the singularities of $\bar{Z}_{0}^{\prime}$ are only ordinary double points,

vi) the singularities of $\bar{Z}^{\prime}$ are isomorphic to the normalizations of the singularities at the origin defined by the equations of the form

$$
z^{k}=x^{k-q} y
$$

in $\mathbb{C}^{3}$, where $k$ and $q$ are coprime positive integers and $k>q$; moreover, the local irreducible components of $\bar{Z}_{0}^{\prime}$ at the singularit'es are defined by $x=0, y=0$ or $x y=$ 0 (cf. [1, Section 2] and [21, Section 4]),

vii) there is no smooth rational curve in $\bar{Z}_{0}^{\prime}$ which intersect the other irreducible components of $\bar{Z}_{0}^{\prime}$ at 1 or 2 points. Let $\theta_{l}: \bar{Z}^{\prime} \rightarrow \bar{Z}$ be the natural holomorphic mapping such that $\vartheta_{l} \circ \bar{\mu}^{\prime}=\bar{\mu} \circ \theta_{l}$. Then $\theta_{l}$ is a finite holomorphic mapping, $\bar{Z}_{0}^{\prime}=$ $\theta_{l}^{-1}\left(\bar{Z}_{0}\right)$ and

$$
\theta_{l} \mid Z^{\prime}: Z^{\prime} \rightarrow Z \text { is an unramified covering, }
$$


where $Z^{\prime}=\bar{Z}^{\prime} \mid D^{*}$. Let $U$ be a neighborhood of the origin $0, x_{i} \in \bar{Z}_{0}, i=1,2$, and $V_{i}$ neighborhoods of $x_{i}$ such that $V_{i} \subset \bar{Z} \mid U$. Put $U^{\prime}=\vartheta_{l}^{-1}(U)$. Let $x_{i}^{\prime}$ be an arbitrary point of $\theta_{l}^{-1}\left(x_{i}\right)$ and $V^{\prime}\left(x_{i}^{\prime}\right)$ the connected component of $\theta_{l}^{-1}\left(V_{i}\right)$ containing $x_{i}^{\prime}$. It follows from (5.4) and [10, p. 48, Proposition 1.6] that

$$
\inf _{x_{i^{\prime}}} d_{Z^{\prime} \mid\left(U^{\prime}-\{0\}\right)}\left(V^{\prime}\left(x_{1}^{\prime}\right) \cap Z^{\prime}, V^{\prime}\left(x_{2}^{\prime}\right) \cap Z^{\prime}\right)=d_{z \mid(U-\{0\})}\left(V_{1} \cap Z, V_{2} \cap Z\right)
$$

Therefore it suffices to show that $\left(Z^{\prime}, \mu^{\prime}, D^{*}\right)$ with $\mu^{\prime}=\overline{\mu^{\prime}} \mid Z^{\prime}$ is hyperbolically imbedded in $\left(\bar{Z}^{\prime}, \bar{\mu}^{\prime}, D\right)$ along $\{0\}$. Assume that this is false. By vi) any local irreducible component of $\bar{Z}_{0}^{\prime}$ at any point is principal. Hence, as in Howard's proof of Theorem A, we can use Hurwitz's theorem to show that the locally uniform limit of a sequence of non-vanishing holomorphic functions is either non-vanishing or identically 0. By the assumption, the same arguments as in the proof of Lemma (1.6) yield a non-constant holomorphic mapping $f: \mathbb{C} \rightarrow \bar{Z}_{0}^{\prime}$ which is a locally uniform limit of holomorphic mappings $f_{\nu}: D\left(r_{\nu}\right) \rightarrow Z^{\prime}, \nu=1,2, \ldots$, where $r_{\nu} \uparrow \infty$ as $\nu \rightarrow \infty$. We see by vi), vii) and Hurwitz's theorem that $f$ maps $\mathbb{C}$ into an irreducible curve of genus $\geqq 2$, into an elliptic curve minus one point or into $\mathbb{P}^{1}(\mathbb{C})$ minus three points; here it may be only the non-trivial case where $f(\mathbb{C})$ is contained in a singular rational curve $C$ which intersects at least one of the other irreducible components of $\bar{Z}_{0}^{\prime}$ at a point $P$ and has a node $P^{\prime}$. In this case, assume that $\mathbb{P}^{\prime} \in f(\mathbb{C})$. Applying vi) at $P^{\prime}$, one sees that $x \circ f \equiv 0$ and $y \circ f \equiv 0$. Hence $f$ must be constant. This is absurd. Thus we have $P^{\prime} \notin f(\mathbb{C})$. Because of the same reason, we have $P \notin f(\mathbb{C})$. Taking the normalization of $C$, we deduce that $f$ maps $\mathbb{C}$ into $\mathbb{P}^{1}(\mathbb{C})$ minus three points. In any case, we have a contradiction. Q.E.D.

\section{Referemces}

[1] Briskorn, E., Rational Singularitäten komplexer Flächen, Invent. Math., \& (1968), 336-358.

[2] Brody, R., Compact manifolds and hyperbolicity, Trans. Amer. Math. Soc., 235 (1978), 213- 219.

[3] Douady, A., Le problème des modules pour les sous-espaces analytiques compacts d'un espace analytique donné, Ann. Inst. Fourier, Grenoble, 16 (1966), 1-95.

[4] Grauert, H., Mordells Vermutung über rationale Punkte auf Algebraischen Kurven und Funktionenköper, Publ. Math. I.H.E.S., 25 (1965), 131-149.

[ 5 ] Green, M., The hyperbolicity of the complement of $2 n+1$ hyperplanes in general position in $\mathbb{P}_{n}$, and related results, Proc. Amer. Math. Soc., 66 (1977), 109-113.

[6] - Holomorphic maps to complex tori, Amer. J. Math., 100 (1978), 615-620. 
[7] Brody's method in value distribution theory, In Geometric Theory of Several Complex Variables, Lecture Notes, 3\$0, Research Institute for Mathematical Sciences, Kyoto Univ., Kyoto, 1978.

[8] Kalka, M., Shiffman, B. and Wong, B., Finiteness and rigidity theorems for holomorphic mappings, Michigan Math. J., 2\& (1981), 289-295.

[9] Kiernan, P., Hyperbolically imbedded spaces and the Big Picard theorem, Math. Ann., 204 (1973), 203-209.

[10] Kobayashi, S., Hyperbolic Manifolds and Holomorphic Mappings, Marcel Dekker, New York, 1970.

[11] Intrinsic distances, measures and geometric function theory, Bull. Amer. Math. Soc., 82 (1976), 357-416.

[12] - Negative vector bundles and complex Finsler structures, Nagoya Math. J., 57 (1975), 153-166.

[13] - The first Chern class and holomorphic symmetric tensor fields, J. Math. Soc. Japan, 32 (1980), 325-329.

[14] Kobayashi, S. and Nomizu, K., Foundations of Differential Geometry Vol. II, Interscience Tracts in Pure and Applied Math., 15., John Wiley \& Sons, New York-Chichester-Toronto, 1969.

[15] Kobayashi, S. and, Ochiai, T., Meromorphic mappings onto compact complex spaces of general type, Invent. Math., 31 (1975), 7-16.

[16] Lang, S., Higher dimensional Diophantine problems, Bull. Amer. Math. Soc., $₫ \mathbb{Q}$ (1974), 779-787.

[17] Lecure, F., Une caractérisation différentielle des points de Weierstrass hyperelliptiques, $C . R$. Acad. Sc. Paris, 281 (1975), 1043-1045.

[18] Manin, Ju., Rational points of algebraic curves over function fields, Izv. Akad. Nauk. SSSR. Ser. Mat., 27 (1963), 1395-1440.

[19] Mori, S. and Mukai, S., The uniruledness of the moduli space of curves genus 11, Algebraic Geometry, Proc. Japan-France Conference at Tokyo and Kyoto 1982, Lecture Notes in Math., 1016, Springer-Verlag, Berlin-Heidelberg-New York, 1983.

[20] Nishino, $\mathbb{T}$., Nouvelles recherches sur les fonctions entières de plusieurs variables complexes (V) Fonctions qui se réduisent aux polynomes, J. Math. Kyoto Univ., $\mathbb{1 5}$ (1975), 527-553.

[21] Nishino, T. and Suzuki, M., Sur les singularitês essentielles et isolês des applications holomorphes à valeurs dans une surface complexe, Publ. RIMS, Kyoto Univ., 116 (1980), 461-497.

[22] Noguchi, J., A higher dimensional analogue of Mordell's conjecture over function fields, Math. Ann., 25\& (1981), 207-212.

[23] Noguchi, J. and Sunada, T., Finiteness of the family of rational and meromorphic mappings into algebraic varieties, Amer. J. Math., 104 (1982), 887-900.

[24] Riebesehl, D., Hyperbolische komplexe Raume und die Vermutung von Mordell, Math. Ann., 257 (1981), 99-110.

[25] Royden, H. L., Remarks on the Kobayashi metric, Several Complex Variables II (Proc. International Conf., Univ. of Maryland, 1970), pp. 125-137, Lecture Notes in Math., 185, 
Springer Verlag, Berlin-Heidelberg-New York, 1971.

[26] - Holomorphic fibre bundles with hyperbolic fibre, Proc. Amer. Math. Soc., 43 (1974), 311-312.

[27] Samuel, P., Lectures on Old and New Results on Algebraic Curves, Tata Institute of Fundamental Research, Bombay, 1966.

[28] Urata, T., Holomorphic mappings into a certain compact complex analytic space, Tohoku Math. J., 33 (1981), 573-585.

[29] - The hyperbolicity of complex analytic spaces, Bull. Aichi Univ. of Education, XXXI (Natural Sci.) (1982), 65-75. 